\title{
Polypyrimidine tract binding proteins (PTB) regulate the expression of apoptotic genes and susceptibility to caspase-dependent apoptosis in differentiating cardiomyocytes
}

\author{
J Zhang ${ }^{1}$, N Bahi ${ }^{1}$, M Llovera ${ }^{1}$, JX Comella ${ }^{2}$ and D Sanchis ${ }^{\star, 1}$
}

Cardiac morphologic abnormalities in mice deficient for key regulators of the caspase-dependent signaling underscored its role in heart development. However, the mechanisms regulating apoptotic gene expression in the developing heart are unknown. As polypyrimidine tract binding proteins (PTB) determine gene isoform expression during myoblast differentiation and contribute to Apaf-1 translation in cell lines, we investigated whether PTB regulate apoptotic gene expression in differentiating cardiomyocytes. Our results show that PTB are expressed in the embryonic heart and are silenced during development, coinciding with a reduction in the expression of apoptotic genes. Overexpression of PTB in postnatal cardiomyocytes, which express low levels of PTB and apoptotic genes, induced an increase in the amount of pro-apoptotic proteins without affecting abundance of their respective transcripts. Translation of the reporter gene Firefly Luciferase preceded by the $5^{\prime}$-untranslated region of Apaf-1 or Caspase-3 was enhanced by PTB in cardiomyocytes. PTB silencing in fibroblasts induced a decrease of apoptotic protein levels. PTB overexpression in cardiomyocytes induced caspase activity and caspase-dependent DNA fragmentation during ischemia, which is otherwise caspase-independent in differentiated cardiomyocytes. Our results show that PTB contribute to apoptotic gene expression and modulate the susceptibility to caspase activation in differentiating rat cardiomyocytes.

Cell Death and Differentiation (2009) 16, 1460-1468; doi:10.1038/cdd.2009.87; published online 10 July 2009

Apoptotic signaling involves an intracellular proteolytic cascade triggered by environmental stimuli through membrane death receptors, or by internal inputs detected by mitochondria and the endoplasmic reticulum. ${ }^{1}$ In addition to contribute to cell death, some regulators of the apoptotic signaling cascade can also influence cell differentiation. ${ }^{2}$ Increasing evidence shows that apoptotic signaling contributes to heart organogenesis. ${ }^{3-5}$ Blockade of caspase activity with several caspase inhibitors induced alterations in the ventriculoarterial connections in the chick embryo, ${ }^{4}$ whereas deletion of genes coding for upstream regulators of apoptosis such as FADD and cFLIP as well as for executioner caspases in the mouse induced ventricular wall noncompaction and lethality. ${ }^{3,5,6}$ Thus, abnormal expression of apoptotic genes could be involved in some inherited and congenital heart diseases, although many of the underlying mechanisms remain unknown.

Our earlier findings suggested that the apoptotic machinery is expressed in the myocardium during embryonic development and is silenced after birth in different rodent models. ${ }^{7,8}$ Apoptotic gene silencing has been also found in stem cells after cardiac-directed differentiation in vitro. ${ }^{9}$ We hypo- thesized that apoptotic gene silencing in cardiomyocytes is associated with their differentiation and or exit of cell cycle, and we analyzed the expression in the developing heart of several proteins known to regulate apoptotic gene expression, cell cycle progression, and differentiation in other cellular models. We reported earlier that expression of the cell cycle regulators E2F parallels the pattern of apoptotic gene expression in the developing heart. ${ }^{8}$ However, E2F deficiency does not affect apoptotic gene expression in the heart in vivo ${ }^{8}$ despite inducing Apaf-1 expression in cell lines. ${ }^{10}$

Polypyrimidine tract binding proteins 1 and 4 (PTB) are RNA-interacting proteins coded by the same gene that have important functions in RNA maturation and protein translation. ${ }^{11,12}$ Binding to precise pyrimidine-rich motifs in pre-mRNAs, PTB1, and PTB4 proteins induce skipping of the adjacent exon determining isoform gene expression or premature termination of the open reading frame. ${ }^{13,14}$ In addition, PTB contribute to internal ribosome entry segment (IRES)-dependent protein translation, ${ }^{12}$ including trranslation of the pro-apoptotic adaptor Apaf-1. ${ }^{12,15}$ Recently, PTB expression has been shown to be repressed during differentiation in neurons ${ }^{16,17}$ and in $\mathrm{C} 2 \mathrm{C} 12$ myoblasts in vitro, ${ }^{18}$

${ }^{1}$ The Cell Signaling and Apoptosis Group at Institut de Recerca Biomèdica de Lleida (IRBLLEIDA)-Universitat de Lleida, Lleida, Spain and ${ }^{2}$ Institut de Neurociències, Universitat Autònoma de Barcelona, Bellaterra, Spain

*Corresponding author: D Sanchis, IRBLLEIDA-University of Lleida, Av. Rovira Roure 80, 25198 Lleida, Spain.

Tel: + 34 973702215; Fax: + 34 973702213; E-mail: daniel.sanchis@ cmb.udl.cat

Keywords: apoptosis; differentiation; cardiomyocyte; gene expression

Abbreviations: PTB, polypyrimidine tract binding proteins; $\mathrm{PPTB}$, endogenous neural PTB; RRMs, RNA recognition motifs

Received 19.12.08; revised 11.5.09; accepted 04.6.09; Edited by: G Melino; published online 10.7.09 
influencing gene isoform expression. Here, we show that expression of PTB is abundant in the developing rat heart and is progressively repressed after birth, and we characterize the role of PTB in apoptotic gene expression and susceptibility to caspase-dependent apoptosis in differentiating cardiomyocytes.

\section{Results}

Apoptotic proteins are abundant in embryonic rat cardiomyocytes but are scarcely expressed in adult cardiomyocytes (Figure 1a) as reported earlier. ${ }^{8}$ We found that polypyrimidine tract binding proteins PTB1 and PTB4, which control gene expression through mRNA maturation and translation, are abundant in the developing heart but disappear progressively after birth both in rat and mouse (Figure 1b). This fact was associated with the differentiation process of cardiomyocytes as suggested by the inverse correlation between the expression pattern of PTB and cardiac $\alpha$-actin in the myocardium (Figure 1b, bar graph). PTB expression silencing during heart development implied the progressive reduction of the level of their transcripts (Figure 1c).

We had shown earlier that although embryonic cardiomyocytes express high levels of apoptotic genes and readily activate caspases in stress situations, postnatal and adult rat cardiomyocytes express low levels of apoptotic genes and die by caspase-independent mechanisms. ${ }^{7}$ We hypothesized that PTB is involved in apoptotic gene expression in the developing heart and that PTB silencing could contribute to decrease apoptotic gene expression in postnatal myocites. Lentiviral-driven overexpression of the human sequences of PTB1 and PTB4 in P4-5 postnatal cardiomyocytes, which express low levels of PTB and apoptotic genes, induced an increase of apoptotic protein expression (Figure 2). However, PTB expression did not alter the steady-state amount of apoptotic gene transcrips (Figure 3 and data not shown). As expected, expression of either human PTB1 or PTB4 induced a slight decrease in endogenous PTB transcripts (Figure 3, second row from top) and skipping of their target exon 10 in the transcript of endogenous neural PTB (nPTB) ${ }^{19}$ (Figure 3).

In an attempt to identify the posttranscriptional mechanisms involved in PTB-dependent apoptotic gene expression in cardiomyocytes, we performed Luciferase reporter gene assays using bicistronic plasmids in which stabilized Firefly Luciferase $(\mathrm{LucF}+)$ is placed after the $5^{\prime}$-untranslated region (5'-UTR) of Apaf-1 or Caspase-3. In this system, Renilla Luciferase (LuCR) is located at the $5^{\prime}$-end of the bicistronic transcript, it is translated by the cap-dependent machinery

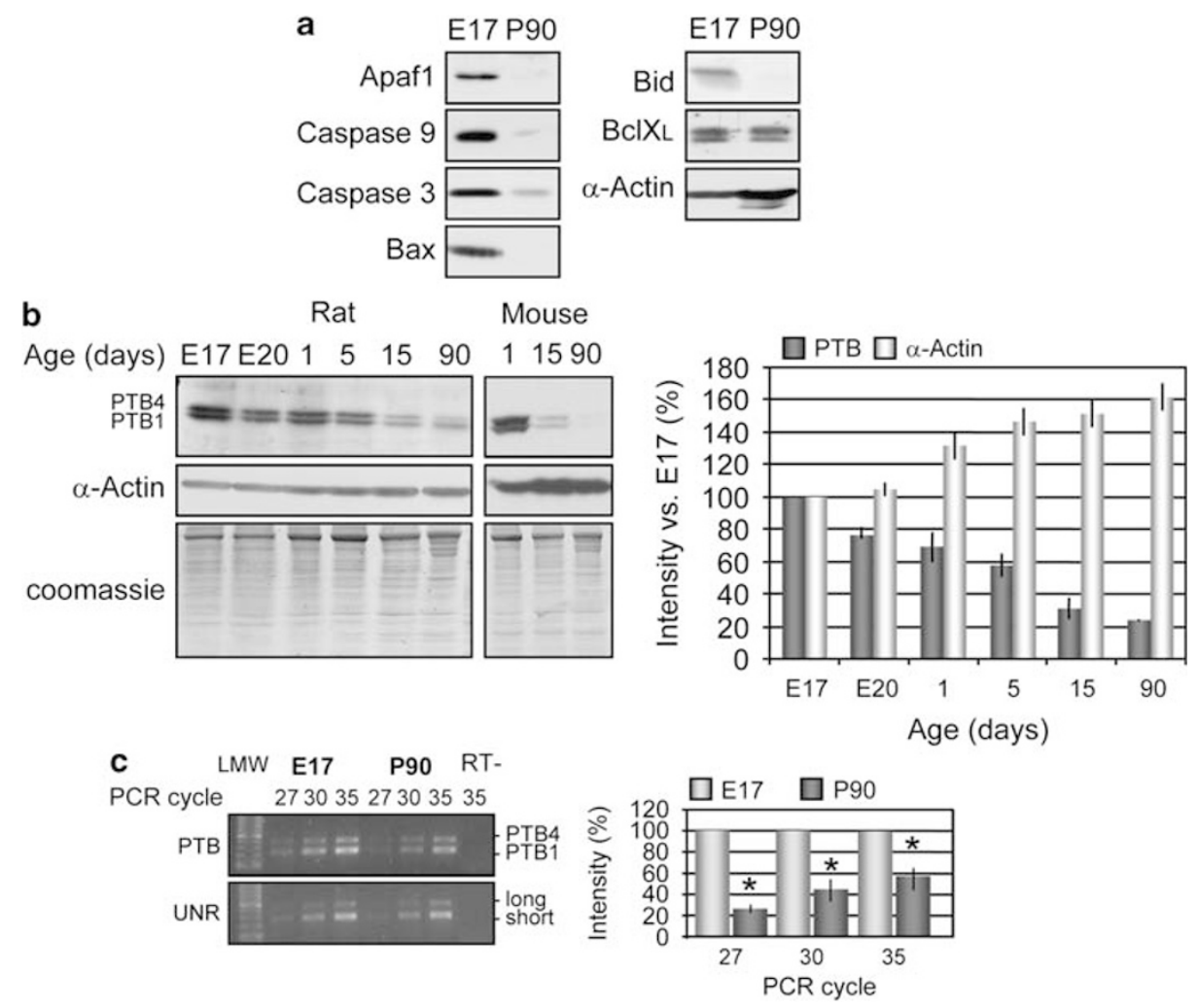

Figure 1 Expression of PTB1 and PTB4 is spontaneously reduced during cardiac development in vivo in parallel to apoptotic gene silencing. (a) Expression of apoptotic proteins in ventricles of embryonic (E17) and adult (P90) rats. (b) Expression of PTB1 and PTB4 proteins in ventricles of rats and mice of different ages from embryonic day 17 (E17) to adult P90. Bar graph: densitometric quantification of western blot signals of PTB and $\alpha$-actin protein expression in rat ventricles $(n=3$, error bars: S.E.M.). Values are referred to the signal at embryonic day 17. (c) Semi-quantitative analysis of PTB1 and PTB4 transcripts in embryonic day 17 (E17) and adult (P90) ventricles. The gel image shows samples obtained at PCR cycles 27,30, and 35 for PTB and UNR. Densitometric analysis of the sum of PTB 1 and 4 bands normalized to E17 values is shown for each PCR cycle; ${ }^{*} P<0.001$ versus E17 signal at the same PCR cycle. In the RT- sample, the reverse transcription reaction was omitted. UNR (upstream of $n$-Ras, Cold shock domain-containing protein E1 (CSDE1)) transcript was amplified as loading control as described earlier ${ }^{7}$ 
a
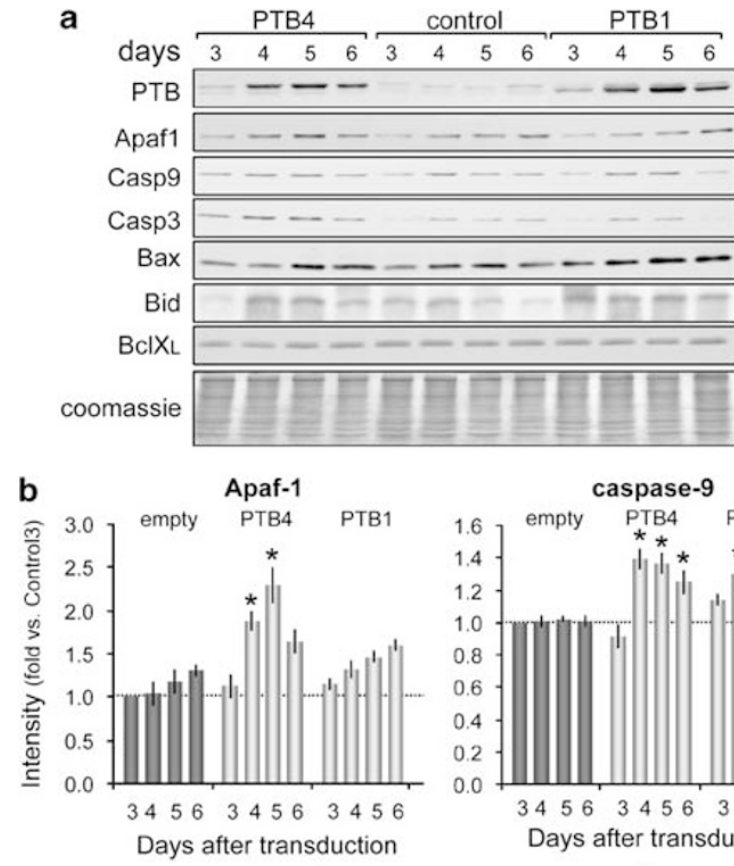
caspase-3

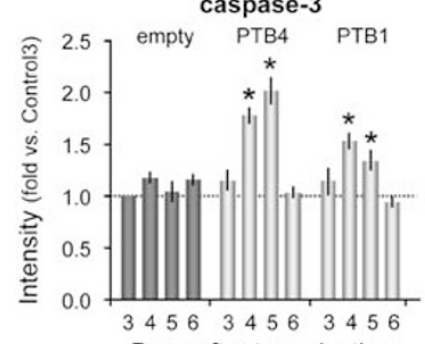

Days after transduction

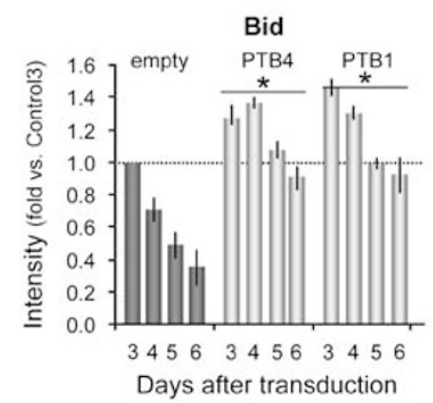

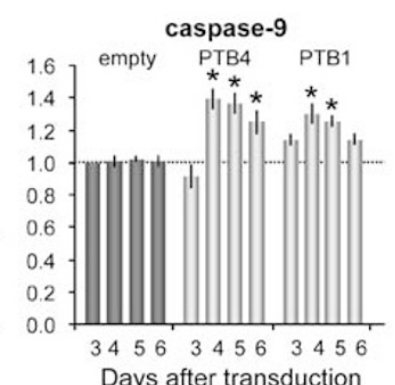

Days after transduction

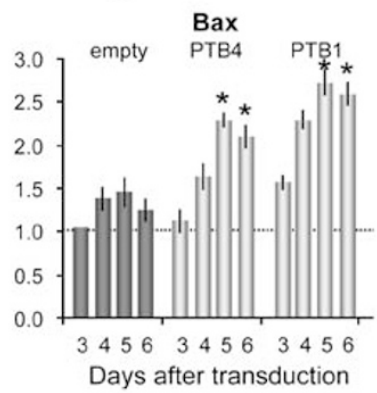

$\mathrm{BCIXL}$

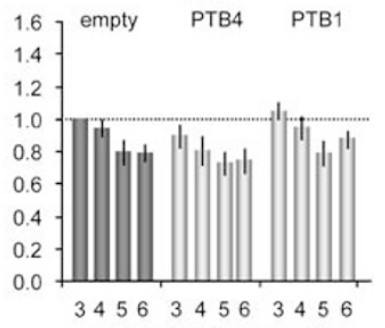

Days after transduction

Figure 2 Overexpression of PTB1 or PTB4 in cultured neonatal cardiomyocytes induces an increase of apoptotic protein expression. (a) Analysis of expression of PTB and apoptotic proteins in P4-5 postnatal rat cardiomyocytes transduced with empty lentiviruses (control) or viral particles inducing expression of human PTB1 or PTB4 at days 3 to 6 after transduction. (b) Densitometric analysis of the signals from three to four independent experiments is expressed as fold change versus controls, at day 3 after transduction \pm S.E.M., ${ }^{*} P \leqslant 0.05$ versus empty vector at the same day

and it is used as a control of transcription/translation of the bicistronic mRNA. This system has been used for studying IRES-dependent translation in cell lines. ${ }^{12,15}$ We transfected the bicistronic plasmids in postnatal cardiomyocytes expressing normal-low levels of PTB and cardiomyocytes overexpressing PTB, and analyzed Renilla and Firefly Luciferase activities 4 days later (see Materials and Methods). High PTB

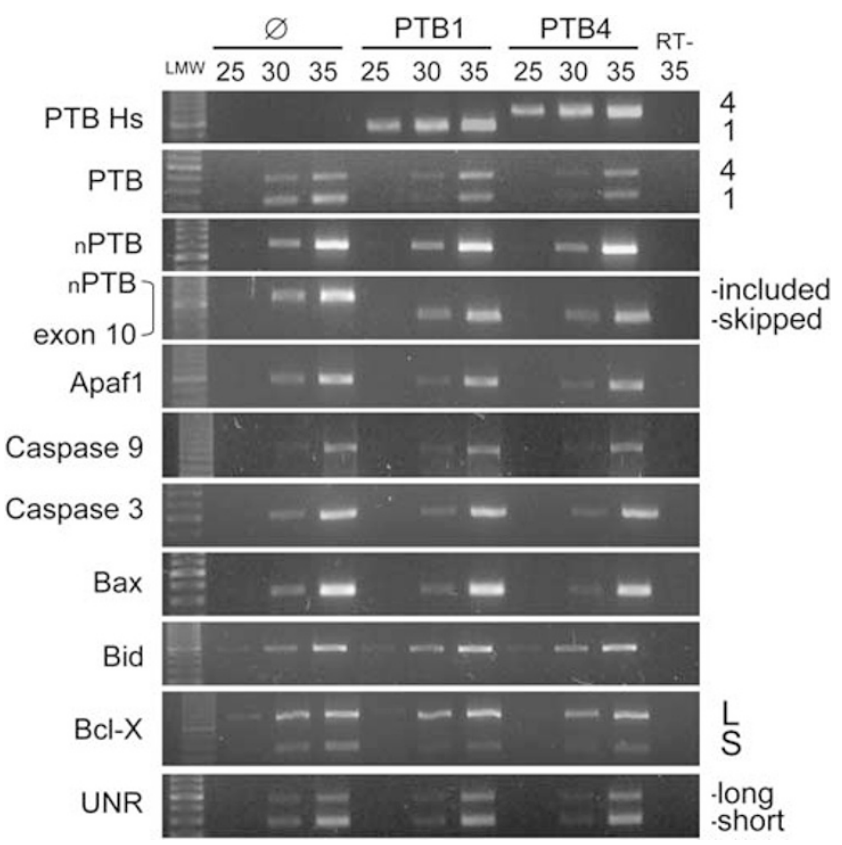

Figure 3 Overexpression of PTB1 or PTB4 in cultured neonatal cardiomyocytes does not affect the level of apoptotic gene transcripts. Steady-state transcript amounts were reverse-transcribed from total RNA extracts of postnatal cardiomyocytes transduced for 4 days with empty $(\varnothing)$, PTB1 or PTB4 vectors, and the CDNAs were PCR-amplified in $50 \mu \mathrm{l}$ reaction volumes. A measure of $10 \mu \mathrm{l}$ were sampled at PCR cycles 25,30 , and 35 and electrophoresed in agarose gels (see Materials and Methods and ref. ${ }^{7}$ ). We used primers specific for human (Hs) and rat PTB 1 and 4, primers detecting the long and short UNR transcripts, as well as primers sensitive to the inclusion/exclusion of nPTB exon 10 (see details in Supplementary Table 1). LMW: ladder of molecular weights; RT- sample: the reverse transcription reaction was omitted (cycle 35 is shown). UNR transcript was analyzed as loading control $\left(\right.$ ref. $\left.^{7}\right)$. Similar results were obtained in three independent experiments

expression in cardiomyocytes induced an increase of LucF + activity controlled by the $5^{\prime}$-UTR of Apaf- 1 or Caspase-3, when normalized to the values obtained in cardiomyocytes transfected with a bicistronic plasmid without any $5^{\prime}$-UTR (Figure 4). LucR activity was similar in all the cell extracts, confirming similar transfection rates and comparable transcription and cap-dependent translation activities. These results were in agreement with the induction of translation of apoptotic genes by PTB. Taken together, the above results show that PTB can induce apoptotic protein expression in differentiating cardiomyocytes without affecting the transcript levels of these genes, and our reporter gene experiments suggest that IRES-dependent translation could be involved in this event.

In addition to mediate IRES-dependent translation, PTB interacts with intronic regions of pre-mRNAs in the nucleus through four RNA recognition motifs (RRMs). To investigate whether the nuclear pool of PTB contributed to apoptotic gene expression, we prepared PTB mutants lacking one or more basic residues in the $\mathrm{N}$-terminus that had been identified as important for nuclear localization using PTB-CAT fusion expression assays. ${ }^{20}$ However, these mutations did not prevent accumulation of PTB in the nucleus of cardiomyocytes (Supplementary Figure 1). Then, we 


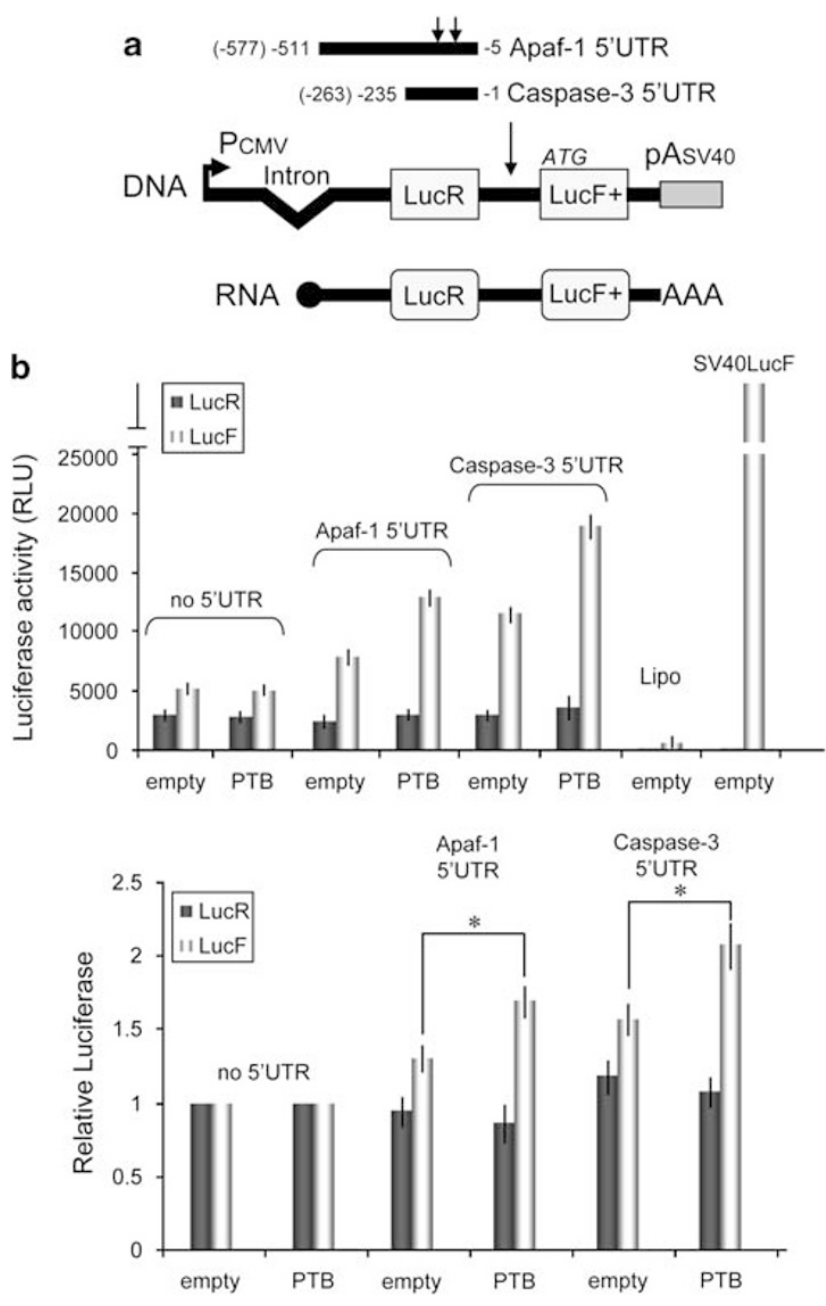

Figure 4 The $5^{\prime}$-untranslated region $\left(5^{\prime}\right.$-UTR) of the transcripts of Apaf- 1 and Caspase-3 drive IRES-dependent translation mediated by PTB in cardiomyocytes. (a) Scheme of the bicistronic reporter constructs. Numbers indicate the distance from the ATG $(+1)$ of the cloned fragments and numbers within parentheses correspond to the $5^{\prime}$ uppermost nucleotide found in the GenBank entry for each transcript (see Materials and Methods). Arrows in the Apaf-1 $5^{\prime}$-UTR indicate location of two known PTB binding motifs. ${ }^{15}$ LucR: Renilla Luciferase; LucF + : stabilized Firefly Luciferase. Transcription is driven by the Cytomegalovirus promoter $\left(\mathrm{P}_{\mathrm{CMV}}\right)$ and finishes with the poly-A tail from the SV40 transcript $\left(\mathrm{pA}_{\mathrm{SV} 40}\right)$. (b) Luciferase activity was measured in cardiomyocytes transduced with empty viruses (empty) or viral particles inducing PTB expression and transfected with the bicistronic plasmids carrying the reporter LucF + gene downstream of the $5^{\prime}$-UTR of Apaf-1 or Caspase-3. pSV40LucF, carrying the LucF gene downstream of the SV40 promoter, was used as a positive control for LucF activity and negative control for LucR activity; pCRHL, which contains a small hairpin sequence between LucR and LucF + was used as positive control for LucR and negative control for LucF. Cardiomyocytes treated with Lipofectamine alone (Lipo) were used as background control. LucR activity allows estimating transcription of the bicistronic construct and cap-dependent translation and was used to confirm similar transfection and transcription rates. LucF + activity takes place only if the cloned $5^{\prime}$-UTR drives IRES-dependent translation. Upper graph: representative experiment showing Luc activity of duplicate samples (RLU: relative luciferase units). Lower graph: Values are expressed as mean \pm S.E.M. Luciferase activity normalized to $\mathrm{pCRHL}$ values from four experiments. $* P \leqslant 0.05$

prepared and transduced a short form of both PTB1 and PTB4 starting at Met175. Short PTB lacked the N-terminal nuclear localization signal (NLS) and the first RRM, which has been shown to influence nuclear shuttling ${ }^{21,22}$ and to be dispensable for RNA binding. ${ }^{21}$ Unexpectedly, although PTB short forms localized to the cytosol, an important pool was still directed to the nucleus (Figure 5a). PTB short forms retained the capacity to induce nPTB exon 10 skipping (Figure 5b) and to enhance expression of Apaf- 1 and Caspase-3 but were not efficient to sustain Caspase-9, Bax, and Bid expression (Figure 5c). Although these results cannot determine whether nuclear PTB contribute to the expression of apoptotic genes, they suggest that the NLS and/or the first RRM are required to sustain Caspase-9, Bax, and Bid expression yet are dispensable for expression of other apoptotic genes. Furthermore, our results show that there are other factors in addition to the NLS and RRM1 contributing to the nuclear localization of PTB, which could have been missed in studies using fusion proteins and cell lines, as discussed below.

To investigate whether forced reduction of PTB expression would weaken apoptotic gene expression, we initially attempted to repress PTB expression through lentiviral-driven shRNA in embryonic E17 cardiomyocytes cultured as reported earlier, ${ }^{7}$ which express high levels of PTB and apoptotic genes. However, expression of apoptotic regulators and other genes were spontaneously reduced in these cultures before the period required for achieving efficient silencing in neonatal cells (i.e. $4-5$ days), precluding further insight in these cells (data not shown). Then, we repressed PTB expression with two independent PTB-specific shRNA constructs in Rat1 fibroblasts, which express high levels of PTB and apoptotic genes, compared with neonatal cardiomyocytes. Decreased PTB expression down to the $20 \%$ induced a slight yet consistent decrease in pro-apoptotic protein amounts (Figure 6), confirming that apoptotic gene expression can be modified by altering endogenous PTB activity.

To ascertain the biological relevance of the global increase of apoptotic gene expression mediated by PTB in cardiomyocytes, we treated postnatal rat cardiomyocytes transduced with empty viruses, PTB1 or PTB4 to experimental ischemia and measured executioner caspase activity, caspase-3 activation, and DNA integrity. Executioner caspase activation during ischemia in PTB1- and PTB4-overexpressing cardiomyocytes was significantly induced, contrary to wild-type cells both measured through enzymatic analysis and immunofluorescence (Figure 7). DNA damage was caspase-independent in cardiomyocytes transduced with empty vector, as reported earlier. ${ }^{7}$ However, ischemia-induced caspase activation in PTB-overexpressing cells correlated with the induction of caspase-dependent, Z-VAD-inhibitable, high molecular weight DNA fragmentation (Figure 8). These results confirmed that global activation of apoptotic gene expression mediated by PTB in differentiating cardiomyocytes contributed to their susceptibility to the induction of caspasedependent apoptosis.

\section{Discussion}

We have shown here that PTB1 and PTB4 are expressed in the murine myocardium during embryonic cardiac development and are down-regulated progressively after birth, after a temporal pattern similar to that of apoptotic gene expression. 
Restoring PTB expression in postnatal cardiomyocytes was sufficient to increase apoptotic protein expression, probably involving IRES-dependent translation, and to induce a shift from caspase-independent to caspase-dependent DNA damage during experimental ischemia, which is caspaseindependent in wild-type postnatal cardiomyocytes. To our knowledge, this is the first report on the mechanisms of regulation of apoptotic gene expression in the developing heart.

Participation of the apoptotic signaling in heart development ${ }^{3,5,6}$ and silencing of this pathway during cardiomyocyte terminal differentiation in vivo ${ }^{7,8}$ and in vitro, ${ }^{9}$ suggest its strict regulation during development in the myocardium. However,

a
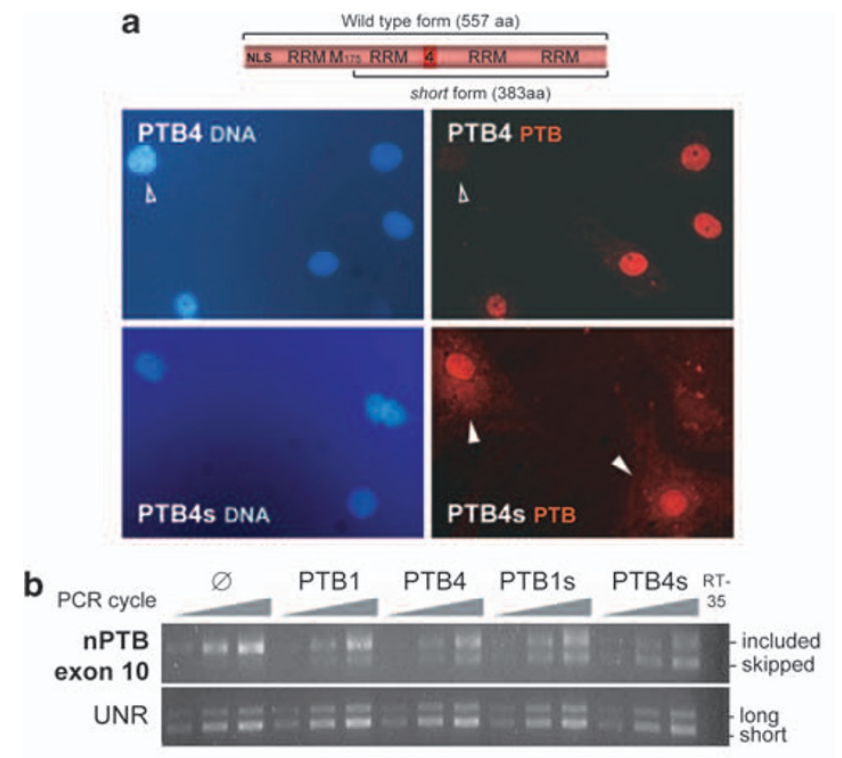

short form (383aa)

c
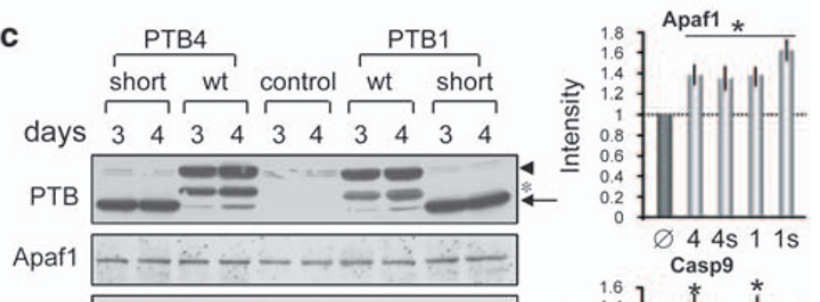

Casp9
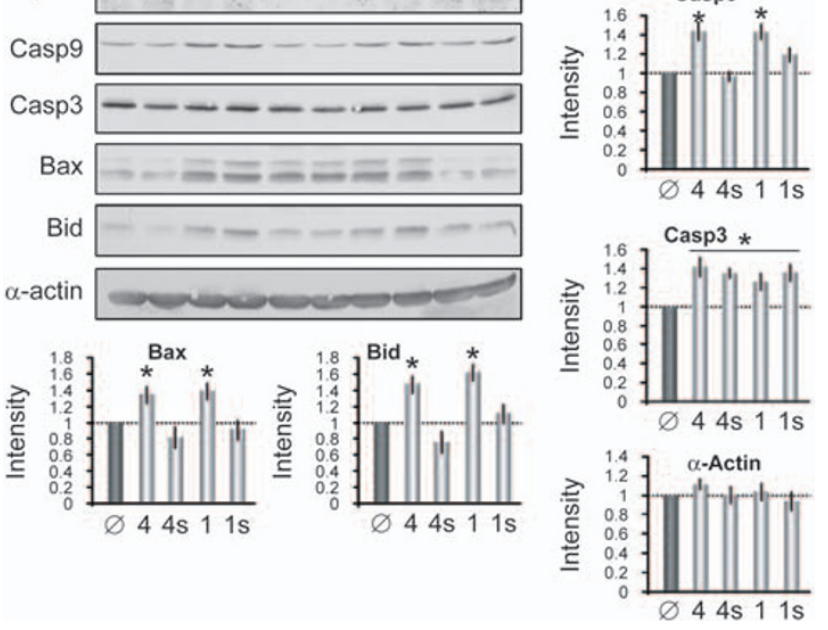

the mechanisms regulating apoptotic gene expression during cardiac development are unknown. Indeed, although gain of function or inactivation of many genes have been reported to induce alteration of the normal rate of cardiomyocyte apoptosis in the developing heart, the possible involvement of changes in the expression of apoptotic genes in these phenotypes was not assessed.

Despite the involvement of PTB proteins in alternative splicing of several genes relevant for cardiomyocyte function such as $\alpha$-actinin, ${ }^{23} \alpha$-Tropomyosin, ${ }^{24} \beta$-Tropomyosin, ${ }^{25}$ and Troponin-T, ${ }^{26}$ as well as the role of PTB in the control of IRESdependent translation of several genes including pro-apoptotic Apaf $1,{ }^{15}$ there is no information about PTB expression and activity neither in mammalian cardiomyocytes nor in heart development. PTB has been suggested to be abundant in the adult chicken heart, ${ }^{27}$ yet our results show that both rat and mouse myocardium spontaneously silence PTB expression early after birth.

Our observation that PTB1 and PTB4 are abundant in the developing embryonic heart and are silenced postnatally suggested to us their role in the control of gene expression during cardiomyocyte differentiation, in a similar way to what has been described in neurons ${ }^{16,17}$ and in differentiating C2C12 myoblasts in vitro. ${ }^{18}$ PTB gain-of-function experiments presented here strongly suggest that PTB contribute to the posttranscriptional regulation of apoptotic gene expression and, hence, susceptibility to apoptosis in differentiating cardiomyocytes. Interestingly, the expression of the antiapoptotic gene $\mathrm{Bcl}-\mathrm{XL}$, unaffected by cardiomyocyte differentiation (Figure 1 and ref. $^{8}$ ), was not modified by PTB. We wanted to identify the mechanisms mediating apoptotic gene expression driven by PTB in cardiomyocytes. PTB has been shown to contribute to Apaf-1 IRES-mediated translation in cell lines. $^{12,15}$ To show this, authors used bicistronic vectors in which the Renilla Firefly (LucR) ORF and the Firefly

Figure 5 PTB lacking the NLS and RRM1 are partially expressed in the nucleus and induce nPTB exon 10 skipping, yet their capacity for inducing apoptotic gene expression is perturbed. (a) Immunofluorescence of postnatal cardiomyocytes overexpressing a short form of PTB lacking the NLS and RRM1 motifs. Images in the figure depict cardiomyocytes overexpressing PTB4 or PTB4 short (identical results were obtained with PTB1/PTB1s, data not shown). DNA was stained with Hoechst dye and PTB expression was immunodetected with an anti-PTB antibody against the c-terminus peptide. Empty arrowhead shows a nontransduced cell where PTB expression is undetectable. White arrowheads show cytosolic PTB expression. (b) Semi-quantitative detection of endogenous steady-state nPTB transcript with primers amplifying the exon10 region in reverse-transcribed total RNA extracts from postnatal cardiomyocytes transduced with PTB1, PTB4, PTB1 short, PTB4 short, or empty $(\varnothing)$ vectors. A measure of $10 \mu \mathrm{l}$ samples from different PCR cycles were electroforesed (see Materials and Methods). UNR is used as loading control. RT- sample: the reverse transcription reaction was omitted (cycle 35 is shown). Similar results were obtained in three independent experiments. (c) Analysis of the expression of PTB and apoptotic proteins in P4-5 postnatal rat cardiomyocytes transduced with empty lentiviruses (control) or viral particles inducing expression of human wild-type (wt) PTB1, PTB4 or their respective short forms, at days 3 and 4 after transduction. Arrowhead: full-length PTB; arrow: short PTB; *: the intermediate band is probably a product of PTB processing. Densitometric analysis of the western blot signals from three to four independent experiments is expressed as fold versus control $(\varnothing)$. 1, PTB1; 1s, PTB1 short; 4, PTB4; 4s, PTB4 short. Values are mean corresponding to day four \pm S.E.M.; ${ }^{\star} P \leqslant 0.05$ versus control 


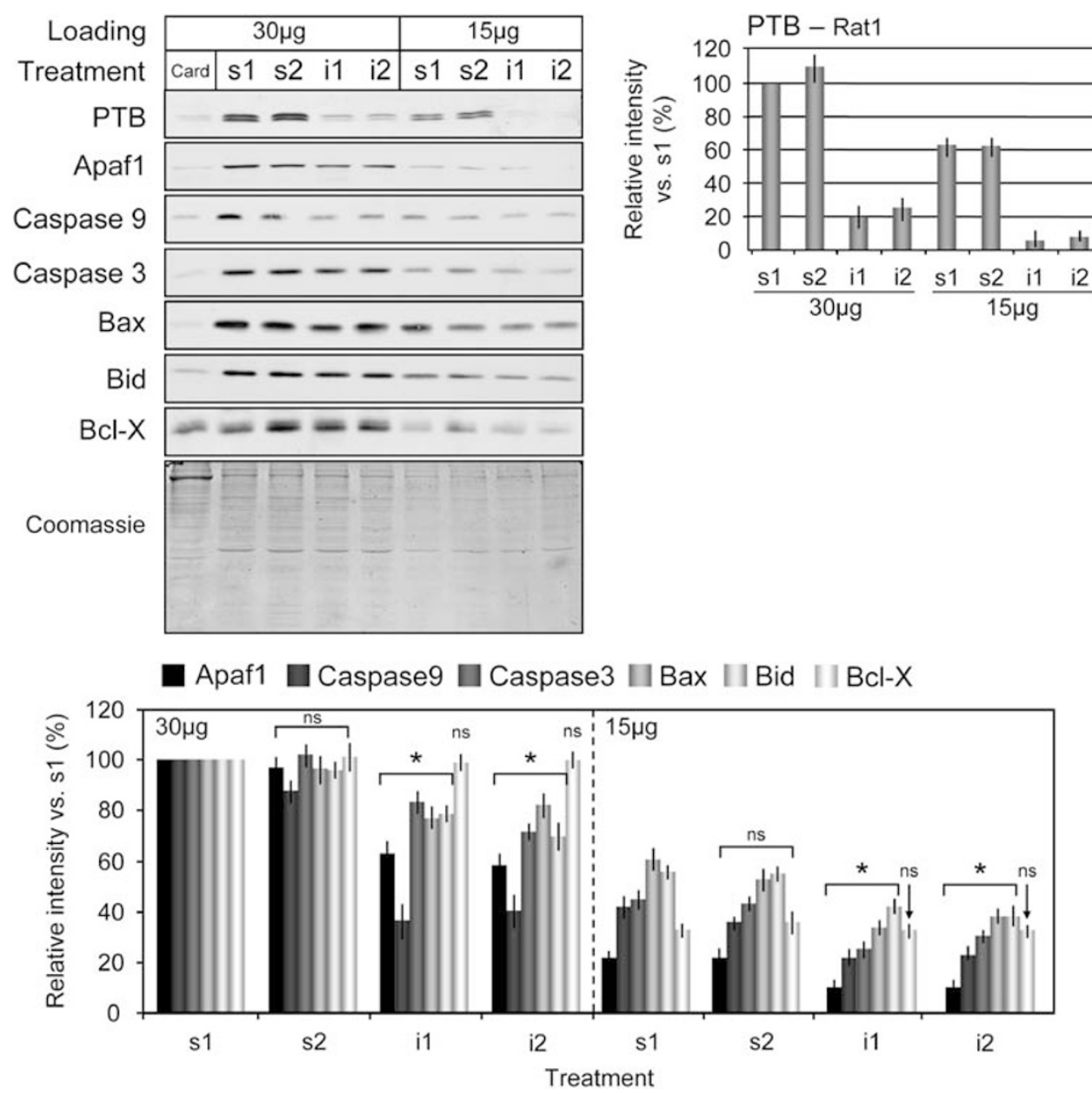

Figure 6 PTB knockdown in Rat-1 fibroblasts induces a general reduction of apoptotic gene expression. Analysis of the expression of PTB and apoptotic proteins in control P4 rat neonatal cardiomyocytes and Rat1 fibroblasts at day four posttransduction with two different shRNA constructs for PTB silencing (i1, i2) or scrambled vectors (s1, s2). In all, 30 and $15 \mu \mathrm{g}$ of protein lysates were loaded for estimation of the response of the antibodies. Small graph: Densitometric analysis for PTB protein signals expressed normalized to $s 1$ from three independent experiments ( \pm S.E.M.). Lower graph: Mean densitometric values of apoptotic protein signals in three to four independent experiments, calculated as described in the Materials and Methods section (Loading: left side, $30 \mu \mathrm{g}$ protein; right side, $15 \mu \mathrm{g}$ protein). Statistical significance versus $\mathrm{S} 1$ is shown. ${ }^{*} P \leqslant 0.05$; ns: not significant

Luciferase (LucF) ORF are separated by the $5^{\prime}$-UTR of Apaf-1. In transfected cells, LucR activity was a measure of transcription and cap-dependent translation and LucF activity required IRES-dependent translation, mediated by PTB in that case. The results presented here using similar bicistronic Luciferase reporter gene constructs suggest that PTB can mediate IRES-dependent translation of Apaf-1 and caspase-3 mRNAs in cardiomyocytes.

We also aimed at analyzing apoptotic gene expression in cardiomyocytes with PTB expression restricted to the cytosol in an attempt to exclude their nuclear functions (i.e. mRNA splicing). We transduced postnatal cardiomyocytes, which express low levels of endogenous PTB, with several PTB constructs harboring mutations or small deletions of amino acids of the $\mathrm{N}$-terminus, reported to be relevant for nuclear targeting in cell lines. ${ }^{20,21}$ However, neither single mutation nor mutation of all key residues altered significantly PTB targeting to the nucleus. This suggested that other regions in PTB are important for nuclear expression in primary cardiomyocytes. We decided to delete both the NLS and first RRM because RRM1 has been shown to be important for nuclear localization ${ }^{21,22}$ and for protein-protein interactions ${ }^{28}$ yet dispensable for PTB-RNA interactions. ${ }^{21}$ This short PTB construct conserved the primary determinant for RNA binding specificity, which resides in RRM3 and RRM4, as shown in Vero and HeLa cell lines, ${ }^{21,29}$ and the primary contributor to dimer stabilization, which resides in RRM2. ${ }^{21,29}$ Unexpectedly, transduction of PTB lacking the NLS and RRM1 in cardiomyocytes induced PTB expression in the cytosol at some extent, yet most of the exogenous PTB was still directed to the nucleus and induced nPTB exon 10 skipping. Nuclear localization of PTB lacking the NLS and RRM1 in primary cardiomyocytes was in apparent contradiction with earlier reports. Indeed, PTB-GST constructs lacking the NLS and RRM1 had been reported to be expressed exclusively in the cytosol of Vero monkey cells, ${ }^{21}$ whereas fussing an $\mathrm{N}$-terminus PTB fragment to CAT was sufficient to direct its expression to the cytosol in 3T3 cells, and deletion of GTK or 
a

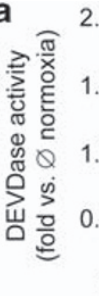

PTBov.

Ischemia

Caspase

Inhibitor

b
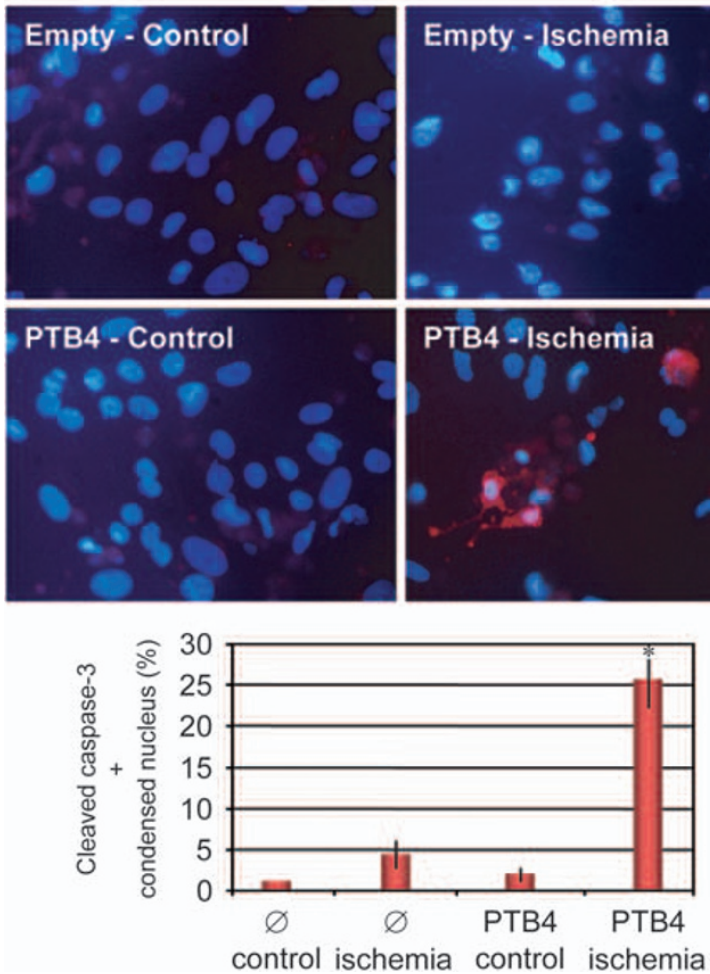

Figure 7 Gene expression changes induced by PTB overexpression in postnatal cardiomyocytes induce a switch from caspase-independent to caspasedependent apoptosis during experimental ischemia. (a) Enzymatic DEVDase activity (as a measurement of executioner caspase activity) was analyzed in cardiomyocytes transduced with empty $(\varnothing), \mathrm{PTB} 1^{1}$ or $\mathrm{PTB} 4^{4}$ vectors and treated with ischemia (see Materials and Methods) in the presence or absence of the pancaspase inhibitor z-VAD-fmk $(100 \mu \mathrm{M})$. Values from three experiments performed in duplicates are expressed as fold increase versus intra-experiment normoxic cardiomyocytes transduced with empty viruses ( \pm S.E.M.). (b) Immunostaining of activated caspase-3 in wild-type and PTB4-overexpressing cardiomyocytes during ischemia. Representative images produced by overlay of the Hoechst staining (chromatin, blue) and anti-cleaved caspase-3 staining (active protease, red) are shown from three independent experiments performed in duplicates. Caspasedependent apoptosis was quantified by counting cells stained with the activated caspase-3-specific antibody with condensed and or fragmented nucleus and is expressed in percentages as mean \pm S.E.M. from three independent experiments performed in duplicates. Statistical significance of changes versus values of control cardiomyocytes during ischemia was calculated for $(\mathbf{a})$ and $(\mathbf{b})(* P \leqslant 0.05$ versus empty virus)
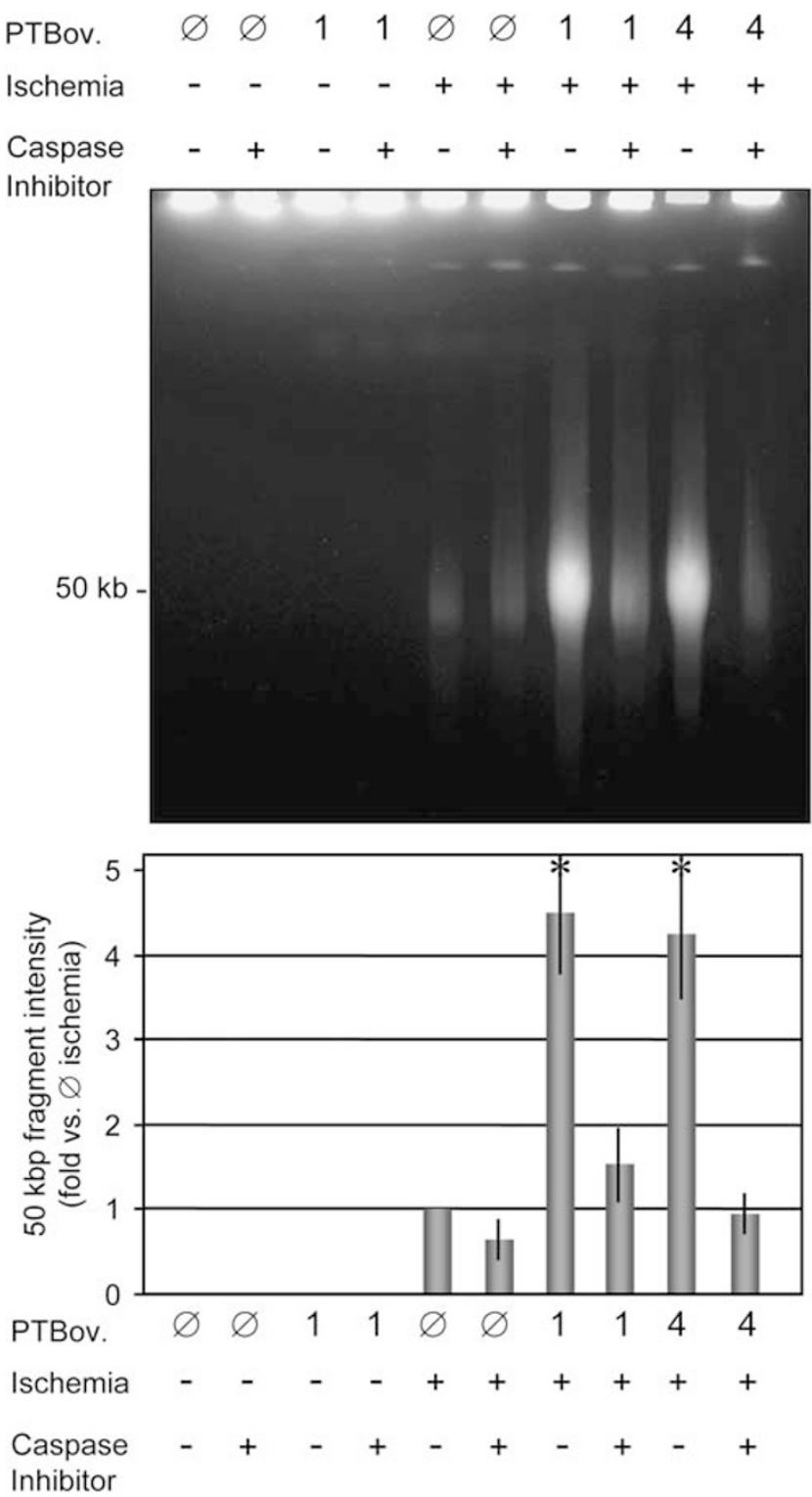

Figure 8 PTB overexpression in postnatal cardiomyocytes induce the activation of caspase-dependent DNA damage during experimental ischemia. High molecular weight DNA fragmentation was analyzed by pulse-field electrophoresis in DNA samples from cardiomyocytes transduced with empty $(\varnothing)$, PTB $1^{1}$ or PTB4 ${ }^{4}$ vectors and cultured in standard conditions or treated with experimental ischemia (see Materials and Methods). Densitometric analysis of the region around the $50 \mathrm{~kb}$ size was performed and values were expressed as fold versus cardiomyocytes transduced with empty viruses and treated with ischemia. Bars represent mean of three independent experiments \pm S.E.M.; $* P \leqslant 0.05$ versus cardiomyocytes transduced with empty viruses in the same experimental conditions

KKF motifs of the $\mathrm{N}$-terminal region restricted CAT expression to the cytosol. ${ }^{20}$ One difference between this study and other's reports, in addition to the use of primary cells instead of cell lines, is that we have analyzed PTB distribution with anti-PTB antibodies and not by detection of a long tag fussed to the protein or a PTB fragment. Our approach implies the possibility of detecting short PTB-induced expression of endogenous full-length PTB that would be directed to the nucleus. However, this was discarded by western blot analysis showing 
no changes in endogenous PTB expression in these experiments. Finally, abnormal behavior of PTB distribution due to overexpression in cardiomyocytes that does not happen in overexpressing cell lines is improbable because, in our hands, overexpression of other genes using identical procedure did not alter their expected subcellular distribution (ref. ${ }^{7}$ and data not shown). Thus, these results suggest that other regions outside the NLS and RRM1 of PTB are relevant for its intracellular localization in cardiomyocytes and/or that PTB can associate with other proteins to enter the nucleus without requiring the NLS. These results also showed that NLS and RRM1 of PTB are important for inducing expression of a subset of apoptotic genes (e.g. Caspase-9, Bax, and Bid), but not for the regulation of other target genes (e.g. Apaf-1 and Caspase-3).

Our results also show that the slight increase in apoptotic gene expression induced by PTB, probably in addition to modifications in the expression of other target genes relevant for apoptosis, is sufficient for changing the main pathway leading to cardiomyocyte damage during stress (i.e. from caspase-independent DNA damage to caspase activation and caspase-dependent DNA damage). These results show that small changes in apoptotic gene expression modify the susceptibility of cardiomyocytes to apoptotic stimuli, and point to PTB as relevant regulators in the control of the susceptibility of differentiating cardiomyocytes to caspase-dependent apoptosis through posttranscriptional regulation of apoptotic gene expression, involving IRES-dependent translation.

\section{Materials and Methods}

Animals, tissues, and cell cultures. The investigation with experimental animals conforms to the Guide for the Care and Use of Laboratory Animals published by the US National Institutes of Health (NIH Publication No. 85-23, revised 1996) and was approved by our Experimental Animal Ethic Committee. Sprague-Dawley rat's hearts were dissected minced into small cubes, rinsed with cold phosphate-buffered saline and snap-frozen into liquid nitrogen. Rat neonata cardiomyocytes were obtained from the heart of 4-5-day-old pups and adult cardiomyocytes were isolated from 3-month-old male rats as described elsewhere. ${ }^{7}$ Rat-1 rat fibroblast cell line was cultured in DMEM medium supplemented with $10 \%$ Fetal Calf Serum (Invitrogen, SA, Spain)

Lentiviral construction and infection. Human PTB1 and PTB4 cDNAs (from Chris W Smith, Cambridge, UK) were cloned into pEIGW vector (from Didier Trono, Geneva, Switzerland) using the Smal and Ndel sites. Using pEIGW-PTB1/4 as templates, short forms of PTB1/4 $\Delta 1-174$ were amplified, sequenced, and cloned into the pEIGW vector in-frame with Smal and Ndel sites. Specific 19 nucleotide sequences were chosen for PTB using the free RNAi design interfaces (see Supplementary Table 1 for details). Primers for small hairpin RNA interference (shRNA) were subcloned into the pLVTHM plasmid as described earlier. ${ }^{7} \mathrm{pEIGW}$ or pLVTHM constructs were transfected into HEK293T cells together with the plasmids psPAX2 and pMD2G (from Didier Trono) to produce viruses. Cells were usually treated after 3 to 6 days of transduction as described elsewhere in detail. ${ }^{7}$

Hypoxic treatment. Experimental ischemia was achieved by culturing cells in Tyrode's solution ( $\mathrm{NaCl} 137 \mathrm{mM}, \mathrm{KCl} 2.7 \mathrm{mM}$, Na2HPO4 8 mM, KH2PO4 $1.5 \mathrm{mM}$ $\mathrm{CaCl} 20.9 \mathrm{mM}$, and $0.5 \mathrm{mM}$, initial pH: 7.2; all reagents from SIGMA-ALDRICH, Spain) inside a Ruskin In Vivo2 400 Hypoxia Workstation (Ruskin, UK) in a mixture of $5 \% \mathrm{CO}_{2}$ and $95 \% \mathrm{~N}_{2}$ following manufacturer's instructions to attain a $0.2 \%$ oxygen concentration. Pan-caspase inhibitor Z-Val-Ala-Asp(OMe)-CH2F (Z-VADfmk) (Merck-Calbiochem) was used at a concentration of $100 \mu \mathrm{M}$.

Western blot, RT-PCR, and immunofluorescence. Protein expression was detected in protein extracts diluted in Tris-buffered $2 \%$ SDS solution at $\mathrm{pH}$ 6.8 as reported earlier. ${ }^{30}$ Antibody data can be found in the Supplementary Table 2.
Each immunodetection was repeated with three to four different membranes containing samples coming from different animals of each age or from cell cultures, as described in each figure legend. Immunoblots were developed with the ECL chemiluminescence detection kit (Biological Industries, Israel). Different exposures were performed on SuperRX Fuji Film (Fuji, Tokyo, Japan) to obtain nonsaturated signals. Densitometry was performed with the Scion Image software (Scion Corporation, Frederick, MD, USA) and qualitative calculations were done comparing data from nonsaturated film images. Special care was taken to assure that antibody dilutions and conditions of immunodetection allowed getting signals in the linear range of antibody response (see Supplementary Figure 2). Total RNA was obtained from frozen tissues or cell pellets with the RNeasy Mini Kit (Qiagen, Spain). RNA concentration measurements and reverse transcription were done as described. ${ }^{7,8}$ Equal volumes of CDNA were amplified by PCR in $50 \mu$ l of reaction mix using a couple of specific primers expanding at least two exons within the gene of interest (see Supplementary Table 1 for details) as described elsewhere. ${ }^{7,8}$ Aliquots $(10 \mu l)$ were taken at PCR cycles 25,30, and 35, mixed with SYBR Safe dye (Invitrogen) and migrated in a $3 \%$ agarose gel. Densitometry of the DNA bands was performed with the Scion Image software (Scion Corporation, Washington, USA) and qualitative calculations were done comparing data from nonsaturated products. Loading was checked by amplification of the UNR transcript. Transcript analyses were performed from three samples coming from different animals and cell cultures. PTB expression was also analyzed by immunofluorescence in two independent cardiomyocyte cultures after fixing in $4 \%$ paraformaldehyde as described earlier ${ }^{7}$ using the antibody against the PTB c-terminal region (see Supplementary Table 2) at 1:5000 dilution. Caspase-3 processing required for its activation was analyzed with a cleaved caspase-3-specific antibody (Cell Signaling, Danvers, MA, USA) following the above procedure. Hoechst staining at the end of the IF procedures was performed to observe nuclear morphology. Each treatment was performed in duplicates in two independent experiments.

Enzymatic caspase activity assay. Executioner caspase enzymatic activity was measured in cell extracts as reported earlier ${ }^{30}$ using the fluorogenic substrate Z-DEVD-AFC (Caspase-3 Substrate IV; Merck-Calbiochem) at $50 \mu \mathrm{M}$ in 96-well plates. Fluorescence was detected with a Biotek Instruments Inc. FL $\times 800$ Fluorescence Reader (Izasa, Spain) with excitation filter set at $360 \mathrm{~nm}$ and emission filter at $530 \mathrm{~nm}$. Data obtained for different experimental conditions were compared within the linear phase of absorbance increase. Data are mean of three independent experiments performed in duplicates.

Luciferase reporter gene assay using bicistronic plasmids. The $5^{\prime}$-UTR region of Apaf-1 and Caspase-3 human genes were PCR-amplified from reverse-transcribed 293T human cell line total RNA, using primers designed from the gene sequences available at GenBank with accession numbers NM_181861 (Apaf-1) and NM_004346 (Caspase-3). Each 5'-UTR was subcloned into the PCRF1AL + bicistronic plasmid ${ }^{31}$ between the Spel and Ncol sites ( $\mathrm{Ncol}$ is the AUG codon of LucF + ). The $5^{\prime}$-UTRs were sequenced to confirm perfect matching with the published sequences. Transcription of the bicistronic transcript is regulated by the CMV promoter and the LucR gene, which lies upstream of the LucF + gene, is translated by a cap-dependent mechanism and is used as control of transfection/ transcription. LucF + translation occurs only if preceded by a sequence mediating IRES-dependent translation. ${ }^{12,15,31} \mathrm{pCRHL}$ vector, harboring a short hairpin sequence between LucR and LucF + was used as positive control for LucR and negative control for LucF, whereas pSV40LucF, where LucF is under the direct control of SV40 promoter was used as a positive control for LucF and negative for LucR. Four hours after seeding, $10 \mu \mathrm{g}$ of bicistronic plasmid was transfected into neonatal cardiomyocyte cultures $\left(0.95 \times 10^{6}\right.$ cells/P35-plate $)$ with Lipofectamine 2000 (Invitrogen) following manufacturer's instructions. A few minutes later, cells were transduced with control lentiviruses or lentiviruses inducing expression of PTB4. Four days later, two cultures per treatment were harvested, washed in cold PBS, lysed in passive lysis buffer and frozen. Quantification of luciferase activity was performed with the Dual-Luciferase Reporter Assay System (Promega, Madison, WI, USA) following manufacturer's instructions and luminescence was detected with a Biotek Instruments Inc. FL $\times 800$ Fluorescence Reader (Izasa, Spain) set at $620 \mathrm{~nm}$ emission wavelength. Values were normalized to the luciferase activity detected in cells transfected with pCRHL. Statistical significance of differences of normalized values coming from four experiments was calculated by the Student's $t$ test.

DNA integrity assay. Cells were pelleted at the end of each treatment and frozen at $-80^{\circ} \mathrm{C}$. Pellets from the same experiment were processed at once. 
They were diluted in $40 \mu \mathrm{l}$ of sterile phosphate-buffered saline, mixed with $40 \mu \mathrm{l}$ of melted $1 \%$ low melting agarose (Sigma) in $0.5 \times$ TBE $(45 \mathrm{mM}$ Tris pH $8.3,45 \mathrm{mM}$ boric acid, 1.0 mM EDTA; all reagents from SIGMA). Each mixture was poured into a block caster and let solidify. Each agarose block was submerged into $1 \mathrm{ml}$ of lysis buffer ( $1 \%$ lauryl sarcosil, $0.5 \mathrm{M}$ EDTA, $10 \mathrm{mM}$ Tris, $\mathrm{pH} 8,100 \mu \mathrm{g} / \mathrm{ml}$ proteinase $\mathrm{K}$; all reagents from SIGMA) at $50^{\circ} \mathrm{C}$ during $24 \mathrm{~h}$ in mild agitation, and rinsed twice with $0.5 \times$ TBE for $1 \mathrm{~h}$ at room temperature. For analysis of DNA high molecular weight degradation, the blocks were then laid into wells of a $1 \%$ agarose, $0.5 \times$ TBE gel (CHEF grade, SIGMA-Aldrich). Pulse field electrophoresis was performed as described earlier ${ }^{7}$ in a CHEF DR-II system (Bio-Rad, Hercules, CA, USA) set to the following protocol: run time, $14 \mathrm{~h}$; switch time from 5 to $50 \mathrm{~s}$; voltage gradient, $6 \mathrm{~V}$ / $\mathrm{cm}$. Gels were stained with SYBR Safe (Molecular Probes, Carlsbad, CA, USA, Invitrogen), visualized by UV exposure and recorded with a Kodak DC290 digital camera. Densitometry was performed as described above.

Statistical analysis. Data are expressed as mean \pm s.e.m. of three or four independent experiments or as specified for each figure. The significance of differences among means was evaluated using the Student's $t$ test. A value of $P \leqslant 0.05$ was considered statistically significant.

Acknowledgements. We greatly appreciate the gift of PTB1 and PTB4 cDNAs and the commentaries from Chris W Smith (University of Cambridge, UK), the gift of the vectors for viral particle production from Didier Trono (École Polytechnique Fédérale de Lausanne, Switzerland) and the gift of bicistronic plasmids from Anne-Catherine Prats (INSERM, Toulouse, France). We give our thanks to Rosa Gómez and Roser Pané (Universitat de Lleida) for their skilled technical assistance. The work was supported by the Ministry of Science and Innovation of Spain (SAF2005-02197 and SAF2008-02271) to DS and Programa de Suport a Grups de Recerca de Catalunya from the Government of Catalonia (AGAUR) (SGR2005-00628) and Ciberned from the Spanish Government to JXC. $\mathrm{JZ}$ is recipient of a pre-doctoral fellowship (FPI program) from the Ministry of Education and Science of Spain. NB was supported by Comissionat per a Universitats i Recerca del Departament de Innovació, Universitats i Empresa from the Government of Catalonia (DIUE) and Fons Social Europeu.

1. Danial NN, Korsmeyer SJ. Cell death: critical control points. Cell 2004; 116: 205-219.

2. Desbarats J, Birge RB, Mimouni-Rongy M, Weinstein DE, Palerme JS, Newell MK. Fas engagement induces neurite growth through ERK activation and $p 35$ upregulation. Nat Cell Biol 2007; 5: 118-125.

3. Yeh WC, Pompa JL, McCurrach ME, Shu HB, Elia AJ, Shahinian A et al. FADD: essential for embryo development and signaling from some, but not all, inducers of apoptosis. Science 1998; 279: 1954-1958.

4. Watanabe M, Jafri A, Fisher SA. Apoptosis is required for the proper formation of the ventriculo-arterial connections. Dev Biol 2001; 240: 274-288.

5. Lakhani SA, Masud A, Kuida K, Porter Jr GA, Booth CJ, Mehal WZ et al. Caspases 3 and 7 : key mediators of mitochondrial events of apoptosis. Science 2006; 311: 847-851.

6. Yeh WC, Itie A, Elia AJ, Ng M, Shu HB, Wakeham A et al. Requirement for Casper (c-FLIP) in regulation of death receptor-induced apoptosis and embryonic development. Immunity 2000; 12: 633-642

7. Bahi N, Zhang J, Llovera M, Ballester M, Comella JX, Sanchis D. Switch from caspasedependent to caspase-independent death during heart development: essential role of endonuclease $G$ in ischemia-induced DNA processing of differentiated cardiomyocytes. J Biol Chem 2006; 281: 22943-22952.

8. Zhang J, Bahi N, Zubiaga AM, Comella JX, Llovera M, Sanchis D. Developmental silencing and independency from E2F of apoptotic gene expression in postmitotic tissues. FEBS Lett 2007; 581: 5781-5786.
9. Doss MX, Winkler J, Chen S, Hippler-Altenburg R, Sotiriadou I, Halbach M et al. Global transcriptome analysis of murine embryonic stem cell-derived cardiomyocytes. Genome Biol 2007; 8: R56.

10. Pediconi $\mathrm{N}$, lanari $A$, Costanzo $A, B$ Blloni $L$, Gallo $R$, Cimino $L$ et al. Differential regulation of E2F1 apoptotic target genes in response to DNA damage. Nat Cell Biol 2003; 5: 552-558.

11. Wollerton MC, Gooding C, Robinson F, Brown EC, Jackson RJ, Smith CW. Differential alternative splicing activity of isoforms of polypyrimidine tract binding protein (PTB). RNA 2001; 7: 819-832.

12. Mitchell SA, Brown EC, Coldwell MJ, Jackson RJ, Willis AE. Protein factor requirements of the Apaf-1 internal ribosome entry segment: roles of polypyrimidine tract binding protein and upstream of N-ras. Mol Cell Biol 2001; 21: 3364-3374.

13. Wagner EJ, Garcia-Blanco MA. Polypyrimidine tract binding protein antagonizes exon definition. Mol Cell Biol 2001; 21: 3281-3288.

14. Wollerton MC, Gooding C, Wagner EJ, Garcia-Blanco MA, Smith CW. Autoregulation of polypyrimidine tract binding protein by alternative splicing leading to nonsense-mediated decay. Mol Cell 2004; 13: 91-100.

15. Mitchell SA, Spriggs KA, Coldwell MJ, Jackson RJ, Willis AE. The Apaf-1 internal ribosome entry segment attains the correct structural conformation for function via interactions with PTB and unr. Mol Cell 2003; 11: 757-771.

16. Boutz PL, Stoilov $P, L i Q$, Lin $C H$, Chawla G, Ostrow K et al, A post-transcriptional regulatory switch in polypyrimidine tract-binding proteins reprograms alternative splicing in developing neurons. Genes Dev 2007; 21: 1636-1652.

17. Makeyev EV, Zhang J, Carrasco MA, Maniatis T. The MicroRNA miR-124 promotes neuronal differentiation by triggering brain-specific alternative pre-mRNA splicing. Mol Cell 2007; 27: 435-448.

18. Boutz PL, Chawla G, Stoilov P, Black DL. MicroRNAs regulate the expression of the alternative splicing factor nPTB during muscle development. Genes Dev 2007; 21: 71-84.

19. Spellman R, Llorian M, Smith CW. Crossregulation and functional redundancy between the splicing regulator PTB and its paralogs NPTB and ROD1. Mol Cell 2007; 27: 420-434.

20. Romanelli MG, Weighardt F, Biamonti G, Riva S, Morandi C. Sequence determinants for hnRNP I protein nuclear localization. Exp Cell Res 1997; 235: 300-304.

21. Pérez I, McAfee JG, Patton JG. Multiple RRMs contribute to RNA binding specificity and affinity for polypyrimidine tract binding protein. Biochemistry 1997; 36: 11881-11890.

22. Kamath RV, Leary DJ, Huang S. Nucleocytoplasmic shuttling of polypyrimidine tractbinding protein is uncoupled from RNA export. Mol Biol Cell 2001; 12: 3808-3820.

23. Southby J, Gooding C, Smith CW. Polypyrimidine tract binding protein functions as a repressor to regulate alternative splicing of alpha-actinin mutually exclusive exons. Mol Cell Biol 1999; 19: 2699-2711.

24. Patton JG, Mayer SA, Tempst $P$, Nadal-Ginard B. Characterization and molecular cloning of polypyrimidine tract-binding protein: a component of a complex necessary for pre-mRNA splicing. Genes Dev 1991; 5: 1237-1251.

25. Mulligan GJ, Guo W, Wormsley S, Helfman DM. Polypyrimidine tract binding protein interacts with sequences involved in alternative splicing of beta-tropomyosin pre-mRNA. J Biol Chem 1992; 267: 25480-25487.

26. Charlet-B N, Logan P, Singh G, Cooper TA. Dynamic antagonism between ETR-3 and PTB regulates cell type-specific alternative splicing. Mol Cell 2002; 9: 649-658.

27. Ladd AN, Stenberg MG, Swanson MS, Cooper TA. Dynamic balance between activation and repression regulates pre-mRNA alternative splicing during heart development. Dev Dyn 2005; 233: 783-793.

28. Kim JH, Hahm B, Kim YK, Choi M, Jang SK. Protein-protein interaction among hnRNPs shuttling between nucleus and cytoplasm. J Mol Biol 2000; 298: 395-405.

29. Oh YL, Hahm B, Kim YK, Lee HK, Lee JW, Song $O$ et al. Determination of functional domains in polypyrimidine-tract-binding protein. Biochem J 1998; 331: 169-175.

30. Sanchis D, Mayorga M, Ballester M, Comella JX. Lack of Apaf-1 expression confers resistance to cytochrome c-driven apoptosis in cardiomyocytes. Cell Death Differ 2003; 10: 977-986.

31. Allera-Moreau C, Delluc-Clavières A, Castano C, Van den Berghe L, Golzio M, Moreau M et al. Long term expression of bicistronic vector driven by the FGF-1 IRES in mouse muscle. BMC Biotechnol 2007; 7: 74 . 